\title{
In situ Spectroscopy of Catalytic Solid-Liquid Interfaces and Chiral Surfaces
}

\author{
Thomas Bürgi*
}

\begin{abstract}
This contribution gives an overview of our recent effort to probe catalytic solid-liquid interfaces in situ and to investigate recognition processes at chiral surfaces. Attenuated total reflection infrared spectroscopy in a dedicated low volume flow-through cell is used to investigate the working catalytic interface. The latter technique is combined with modulation spectroscopy, which relies on the perturbation of the system under investigation by a periodically varying external parameter. A digital phase-sensitive detection results in high quality spectra. The method furthermore yields kinetic information and helps disentangle complex spectra. The described tool is therefore ideally suited for the investigation of complex systems. Applications in the fields of heterogeneous catalysis and recognition at chiral solid-liquid interfaces are presented. Our aim is a better molecular level understanding of these processes and, based on this knowledge, a rational design of better catalyst materials.
\end{abstract}

Keywords: Chiral recognition - Enantioselective catalysis · In situ spectroscopy · Solid-liquid interfaces · Vibrational circular dichroism

\section{Introduction}

Rational design of improved catalyst materials largely relies on a molecular level understanding of the corresponding catalytic reaction [1]. A key information in this respect is the structure of the active site, which is responsible for the catalysis. In recent years it was realized that a catalyst may look quite different at reaction conditions and under conditions far from these. Motivated by this insight much effort has been devoted to the characterization of working catalysts. The correlation of catalytic outcome, such as activity and selectivity, with the state of a catalyst, like oxidation state and composition of the adsorbate layer, yields desired information on the active site and the mechanism of the catalytic reaction. This is the field of in situ or

\footnotetext{
${ }^{*}$ Correspondence: Prof. Dr. T. Bürgi Institut de Chimie

Université de Neuchâtel

Avenue de Bellevaux 51

Case postale 2

CH-2007 Neuchâtel

Tel.: +41327182412

Fax: +41327182511 (secrétariat)

E-Mail: thomas.burgi@unine.ch
}

operando spectroscopy [2-5]. For a heterogeneously catalyzed reaction in situ spectroscopy requires the investigation of an interface. Under conditions relevant to catalysis the powerful electron spectroscopic techniques of surface science are not practical and hence other techniques have to be developed and applied, which are operable at elevated pressures.

In contrast to solid-gas interfaces [6] catalytic solid-liquid interfaces have hardly been investigated, despite their importance. Such interfaces play an important role in Swiss chemical industry with its strength in production of fine chemicals. Here we report on our effort made in the last years in probing these interfaces at work. The methods described below have potential for fundamental investigations in other fields of research where solid-liquid interfaces play an important role, such as electrochemistry [7], tribology [8], environmental chemistry [9], biosensor research and the investigation of biological membranes [10].

\section{Experimental Approach}

Two major challenges encountered in the spectroscopy of a working catalytic sol- id-liquid interface are sensitivity and complexity. The latter has to do with the fact that typically many species can be found simultaneously at such interfaces, ranging from reactants over intermediates to products and spectators, which are not involved in the catalytic cycle. We use attenuated total reflection (ATR) infrared spectroscopy [11] in combination with a dynamic technique, modulation spectroscopy [12][13] to cope with these difficulties. Fig. 1 schematically shows the principle of the ATR technique. An infrared beam from a Fourier transform infrared spectrometer is coupled into an internal reflection element (IRE). The beam is internally reflected inside this element. At the position where the beam is reflected back into the IRE an evanescent electric field forms, which probes a small volume close to the IRE. The penetration depth $d_{p}$ of the field depends on the angle of incidence of the infrared beam, the wavelength of the light and the refractive indices of the IRE $\left(\mathrm{n}_{1}\right)$, the sample $\left(\mathrm{n}_{2}\right)$ and the solution $\left(\mathrm{n}_{3}\right)$ above. Typically the penetration depth is on the order of one $\mu \mathrm{m}$ or less. The real benefit of the technique is the possibility to study adsorbed molecules in the presence of even strongly absorbing solvents. We have applied the ATR technique to 
study model catalysts, which are prepared by physical vapor deposition of thin films onto an IRE [14-20] and real powder catalysts [12][21][22].

\section{Adsorption at Metal-Liquid Interfaces}

Using multiple reflection IREs ATR spectroscopy is a sensitive tool, which allows the study of even weakly absorbing molecules adsorbed on metal surfaces. Using ATR we were able for the first time to investigate the chiral modification of a $\mathrm{Pt}$ catalyst. Fig. 2 shows ATR spectra of cinchonidine, a cinchona alkaloid, adsorbed on a $\mathrm{Pt} / \mathrm{Al}_{2} \mathrm{O}_{3}$ film in the presence of $\mathrm{CH}_{2} \mathrm{Cl}_{2}$ solvent under hydrogenation conditions [15][16]. Such chirally modified surfaces are used as heterogeneous catalysts for the enantioselective hydrogenation of $\alpha$-ketoesters and other ketones [23][24]. The signals reveal a strong dependence on cinchonidine concentration. Analysis of the spectra by comparison with model com- pounds such as quinoline or pyridine and based on $a b$ initio calculations leads to the following picture (Fig. 2): At low concentration cinchonidine adsorbs via the quinoline part of the molecule. $\pi$-Bonding via the aromatic system results in firm anchoring of cinchonidine on Pt. The quinoline ring is oriented predominantly parallel to the surface. Such an adsorption mode was proposed to play the dominant role for enantiodifferentiation, in which intermolecular interactions between cinchonidine and the adsorbed reactant are thought to be crucial [25-28]. At higher cinchonidine concentration the free space on the Pt surface becomes more restricted and two additional species are observed, which are tilted with respect to the surface. In one the hydrogen in $\alpha$-position to the quinoline $\mathrm{N}$ is abstracted. This species is strongly attached to the surface via a Pt-C bond. Such an adsorption mode is not observed on Pd [19]. Finally, a weakly bound cinchonidine species is observed, which is bound via $\mathrm{N}$ lone pair bonding.

\section{Modulation Spectroscopy and In situ Investigations of Catalytic Reactions}

The sensitivity of the ATR method is drastically increased through modulation spectroscopy. The basic idea is given in Fig. 3 . The sample is disturbed by periodically varying an external parameter such as temperature, pressure, reactant concentration or the absolute configuration of a chiral probe molecule. All the species in the system which are affected by this external parameter, will also change periodically at the same frequency as the stimulation or multiples thereof [12]. The absorbance variations $A(\tilde{v}, t)$ are followed by measuring spectra at different times $t_{0}, t_{1}, \ldots t_{n}$ within the modulation period $\mathrm{T}$ (Fig. 3). The time-resolved absorbance spectra are then converted into phase-resolved absorbance spectra by a digital phase-sensitive detection (PSD) according to Eqn. (1):

$$
\begin{aligned}
& A_{k}^{\phi_{k}^{P S D}}(\tilde{v})=\frac{2}{T} \int_{0}^{T} A(\tilde{v}, t) \sin \left(k \omega t+\phi_{k}^{P S D}\right) d t \\
& k=1,2, \ldots
\end{aligned}
$$

Here $\tilde{v}$ is the wavenumber, $\omega$ is the stimulation frequency, $\mathrm{T}$ is the modulation period and $\phi_{\mathrm{k}}{ }^{\mathrm{PSD}}$ is the demodulation phase angle. With one set of time-resolved spectra Eqn. (1) can be evaluated for different phase angles $\phi_{\mathrm{k}}{ }^{\mathrm{PSD}}$ resulting in a series of phase-resolved absorbance spectra. k determines the frequency, at which the timedependent signals $A(\tilde{v}, t)$ are demodulated, i.e. fundamental of the stimulation frequency, first harmonic and so on. The digital PSD is a narrow-band technique and drastically increases sensitivity by typically one order of magnitude. Furthermore, the frequency-dependent phase-lag between stimulation and response ( $\phi$ in Fig. 3) and the amplitude of the response bear detailed kinetic information of the system under investigation. Static signals originating from species that are not affected by the external parameter and that usually dominate the infrared spectra of catalytic solid-liquid interfaces, are completely eliminated by the PSD.

Using this combination of ATR and modulation spectroscopy we investigated the enantioselective hydrogenation of a pyrone over a cinchonidine-modified $\mathrm{Pd} / \mathrm{TiO}_{2}$ catalyst [12]. In this case the catalyst was a supported powder catalyst deposited onto an internal reflection element. Fig. 4 shows the results of a concentration modulation experiment and compares time- and phaseresolved spectra. Most of the signals observed in the time-resolved spectra are static, which means that the species associated with these signals are not affected by the
Fig. 1. Schematic view of ATR principle. Top: Small volume ATR flowthrough cell. Bottom: Enlarged region of the internal reflection element (IRE)-catalyst-solution interface. The penetration depth of the evanescent field $d_{p}$ depends, among other quantities, on the refractive indices $\mathrm{n}$ of IRE, sample and solution and is typically on the order of $1 \mu \mathrm{m}$. 


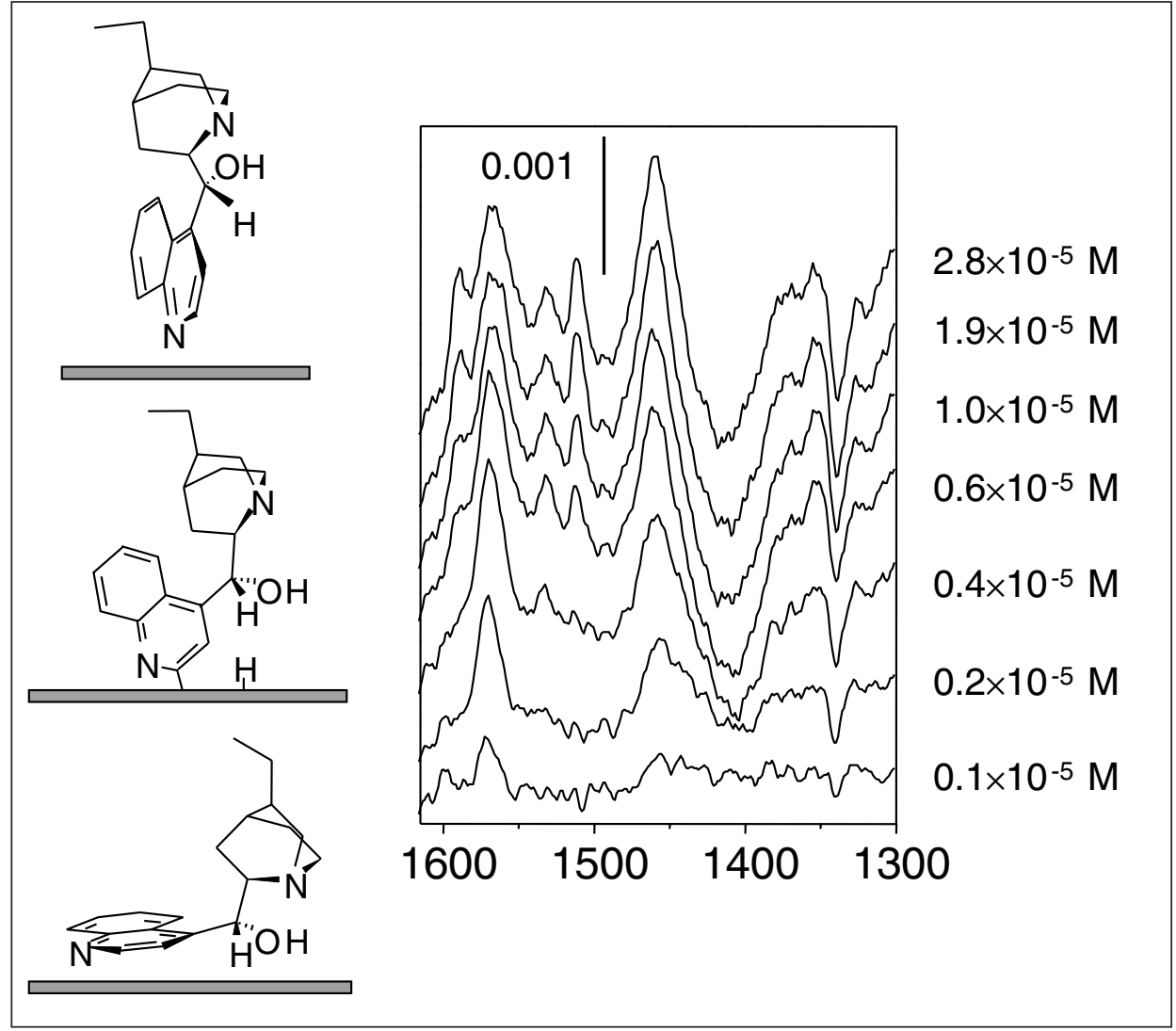

Fig. 2. ATR spectra of cinchonidine adsorbed on $\mathrm{Pt} / \mathrm{Al}_{2} \mathrm{O}_{3}$ at $283 \mathrm{~K}$ recorded while flowing solutions of cinchonidine in hydrogen-saturated $\mathrm{CH}_{2} \mathrm{Cl}_{2}$ at increasing concentration $\left(1 \times 10^{-6}-2.8 \times\right.$ $10^{-5} \mathrm{M}$ ) over the sample. The negative bands at 1420 and $1339 \mathrm{~cm}^{-1}$ are due to solvent decomposition products $\left(\mathrm{CH}_{2}\right.$ and $\left.\mathrm{CCH}_{3}\right)$ displaced from the surface by cinchonidine. The left part of the Fig. schematically shows the three different adsorption modes contributing to the spectra.

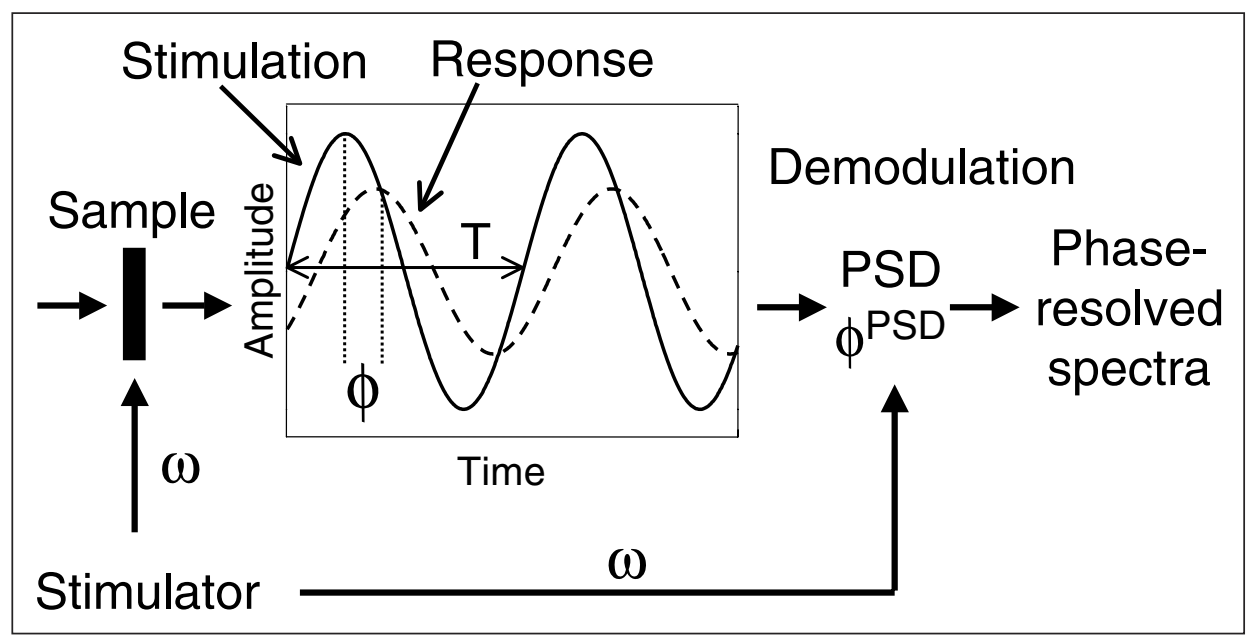

Fig. 3. Principle of modulation spectroscopy. The sample is perturbed by periodically changing an external parameter (stimulation). The concentration of species, which are affected by the external stimulation, and hence the absorbance associated with these species, are periodically changing at the same frequency as the stimulation (response). Time-resolved spectra are recorded within the modulation period $\mathrm{T}$. The signal can be averaged over several modulation periods. The response can be retarded with respect to the stimulation (phase lag $\phi$ ). The timeresolved signals are then demodulated (PSD) at different phase-angles $\phi^{\mathrm{PSD}}$ according to Eqn. (1), which results in a set of phase-resolved spectra. More details are reported in [12]. concentration modulation. The species which respond to the stimulation are revealed in the phase-resolved spectra. Interestingly the latter strongly change as a function of the demodulation phase-angle $\phi^{\mathrm{PSD}}$. This shows that species with distinctly different kinetics contribute to the spectra and allows the discrimination between spectators and species involved in the catalytic cycle. Fig. 5 shows the findings of the study. After the initial fast hydrogenation of a first double bond, the lactone $\mathbf{3}$ is formed in a slower subsequent hydrogenation step. The lactone partly hydrolyses and the resulting carboxylic acid 4 accumulates on the catalyst surface. Analysis of the product mixture collected at the outlet of the ATR cell reveals that the enantiomeric excess (ee) is not constant during the modulation period. The ee is initially high $(75 \%)$ when the pyrone is admitted to the cell and decreases afterwards to a steady state value (Fig. 5). The time behavior of this decay follows the accumulation of the acid on the catalyst surface, which indicates that the acid hinders enantiodifferentiation. We have investigated the interaction of carboxylic acids with cinchona alkaloids by vibrational circular dichroism (VCD) spectroscopy [29]. The latter technique involves the measurement of differential absorption of left- and rightcircularly polarized infrared light by chiral samples [30][31]. In combination with $a b$ initio quantum chemical calculations detailed information on the structure of dissolved chiral molecules can be obtained. We found that the alkaloids form specific interaction complexes with carboxylic acids via two hydrogen bonds involving the protonated quinuclidine $\mathrm{N}$ and the $\mathrm{O}-\mathrm{H}$ group of the alkaloid (for the structure of cinchonidine see Fig. 2). Such an interaction is thought to hinder enantiodifferentiation.

\section{Enantiospecific Interactions at Chiral Solid-Liquid Interfaces}

Chiral interfaces are ubiquitous in nature and play an important role in technology, such as in separation processes [32]. For example there is currently a large interest in a more fundamental understanding of the recognition between chiral selector (chiral stationary phase) and selectand that leads to separation of enantiomers in chromatography [33]. An ideal tool for such investigations would combine surface sensitivity with selectivity for the relevant enantiospecific intermolecular interactions. Unfortunately, many surface sensitive techniques that are susceptible to intermolecular interactions, such as reflection absorption infrared spectroscopy (IRRAS) [34] or sum 


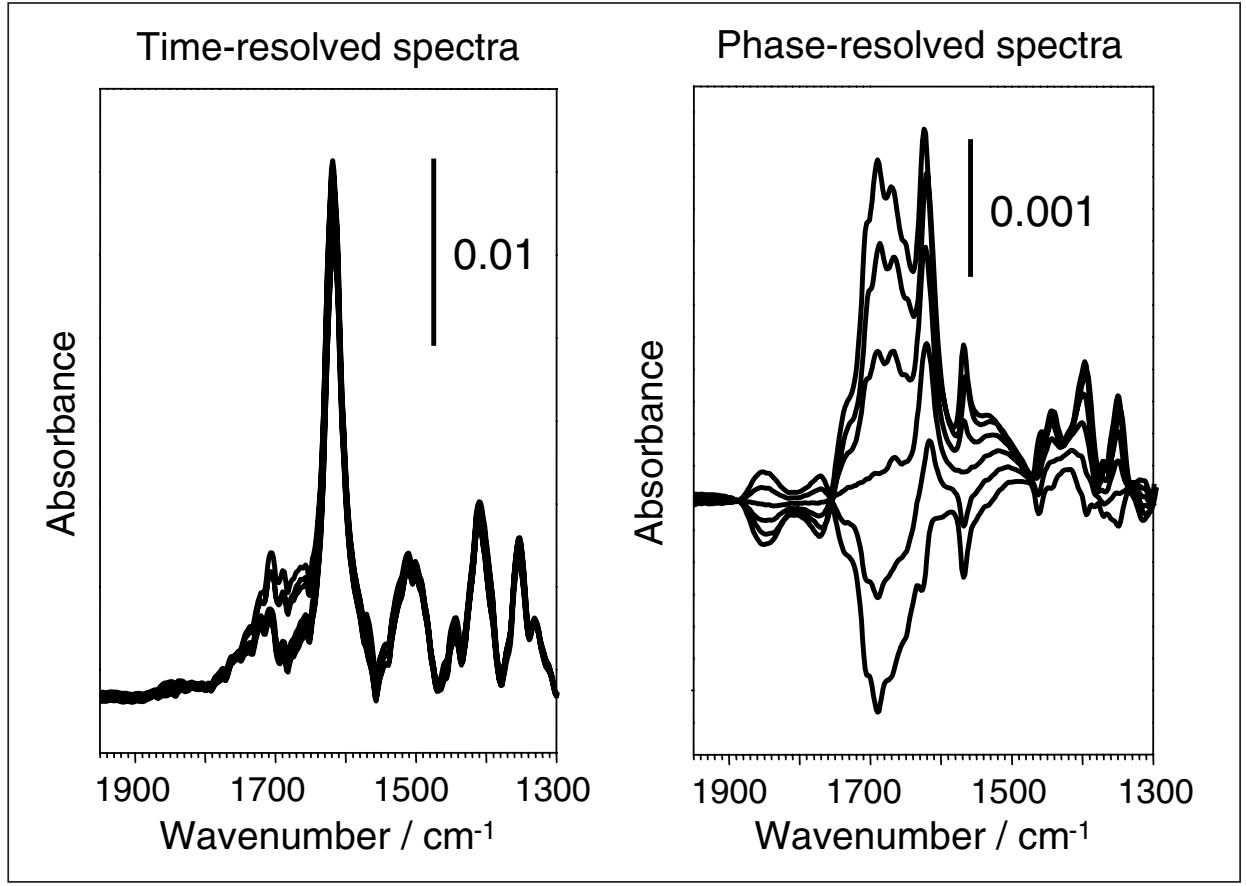

Fig. 4. Selection of time- and phase-resolved ATR spectra (demodulated at different phase angles) of a modulation experiment. The concentration of the pyrone reactant 1 (see Fig. 5) was modulated between 0 and $3.6 \mathrm{mmol} / \mathrm{l}$ with a modulation period of $448 \mathrm{~s}$. Solvent: Hydrogensaturated isopropanol; catalyst: $5 \mathrm{wt} \% \mathrm{Pd} / \mathrm{TiO}_{2}$. For the time-resolved spectra the reference was recorded before starting the modulation. The phase-resolved spectra are independent of the choice of the reference.

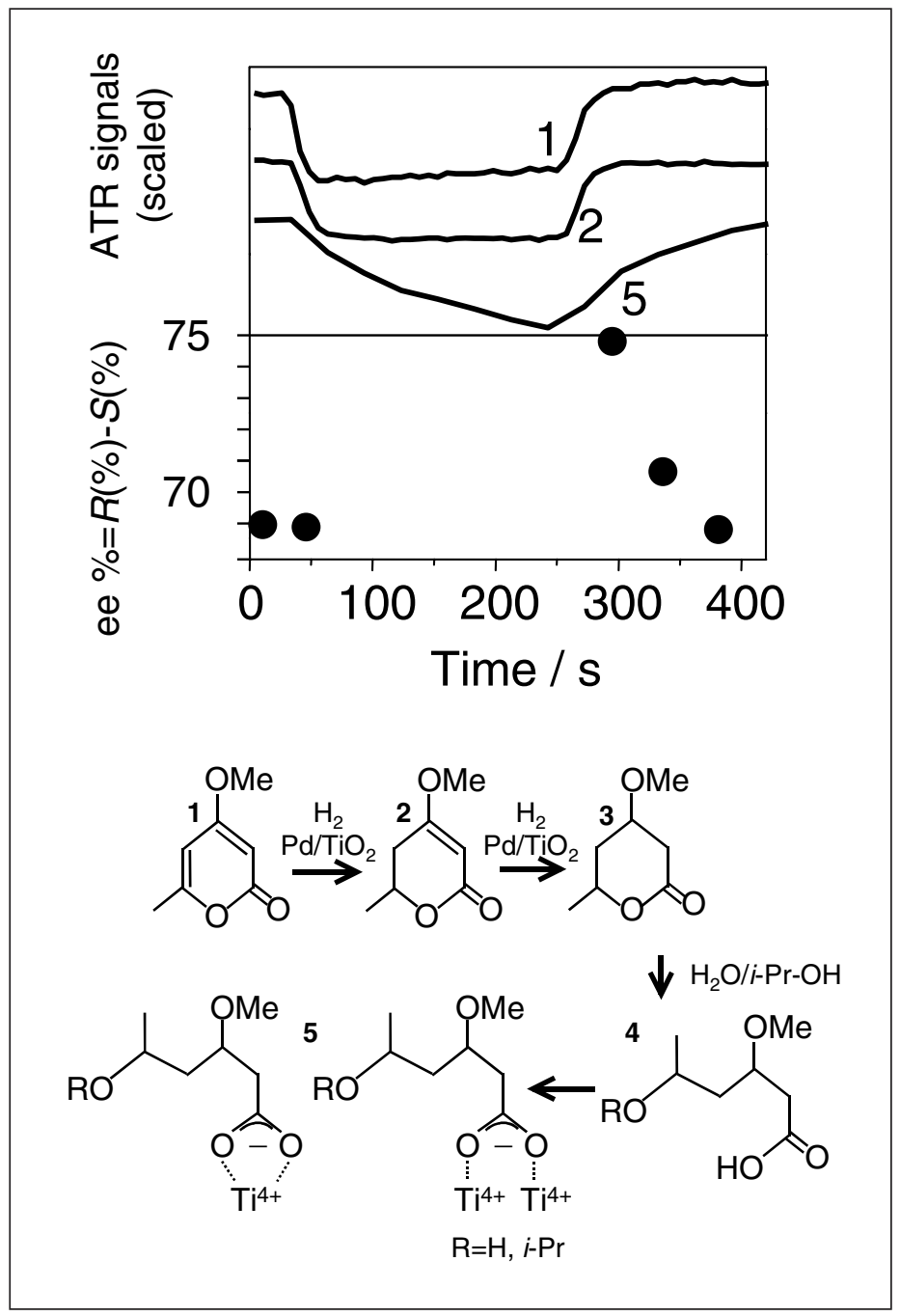

frequency and second harmonic generation (SFG, SHG) [35] do not selectively probe enantiospecific interactions. As a consequence the obtained spectral information is usually strongly congested by signals from non-specific interactions. On the other hand chiroptical methods like vibrational Raman optical activity (VROA) [36] and vibrational circular dichroism (VCD) [30] selectively probe the chiral part of a sample but are not surface sensitive.

We recently demonstrated the potential of a method that combines surface sensitivity with the selectivity to probe enantiospecific interactions at chiral solid-liquid interfaces [37]. The method combines ATR (Fig. 1) and modulation spectroscopy (Fig. 3). The external parameter that is periodically altered is the absolute configuration of a chiral probe molecule (selectand). Signals arising from dissolved species are filtered out because the spectra of enantiomers are identical (to within the resolution of our spectrometers) and the total concentration of both enantiomers does not change during the experiment. More important, non-specific interactions of the enantiomers with the surface are filtered out due to the same reason. Only signals are detected that result from the different diastereomeric interactions of the two enantiomers of the probe molecule with the chiral surface (selector). These differential signals may be small, but they are enhanced by the phase-sensitive detection (see Eqn. (1)).

Fig. 6 shows phase-resolved spectra of two experiments conducted under identical conditions. Two solutions at equal concentration of the opposite enantiomers of ethyl lactate flowed alternatively through the ATR cell (Fig. 1) thus periodically changing the absolute configuration of the lactate. One experiment (top spectra in Fig. 6) was performed in the absence of a chiral sample that could discriminate between the two enantiomers. No significant signals are observed in this case. In the second experiment (bottom spectra) chiral information was present in the form of a chiral stationary phase (CSP), which is amylose tris[(S)$\alpha$-methylbenzylcarbamate] coated onto silica-gel (see Fig. 6). The observed signals arise due to the different diastereomeric interactions of the ethyl lactate enantiomers with the CSP. In particular the spectra show that the amide groups of the chiral stationary phase serve as hydrogen bond donor $(\mathrm{N}-\mathrm{H})$ and acceptor $(\mathrm{C}=\mathrm{O})$. The ester and

Fig. 5. Top: Selected ATR signals as well as enantiomeric excess (ee, solid circles) as a function of time for the experiment shown in Fig. 4. ATR signals are shown of the reactant $\mathbf{1}$, the primary hydrogenation product 2 and carboxylates adsorbed on $\mathrm{TiO}_{2}$ 5. Bottom: Reactions observed by in situ ATR spectroscopy. Reprinted with permission from [12]. Copyright (2002) American Chemical Society. 
Fig. 6. Top: Demodulated ATR spectra at phase angles $\phi^{\mathrm{PSD}}=10^{\circ}, 20^{\circ}$, $30^{\circ} \ldots 90^{\circ}$. The absolute configuration of ethyl lactate $(1.8 \mathrm{mM}$ in hexane) was modulated (modulation period $T=224 \mathrm{~s}$ ). The top spectra were measured in the absence of CSP on the clean Ge IRE, whereas the lower spectra were measured in the presence of CSP under otherwise identical conditions. Bottom: Structure of the CSP, which is amylose tris[(S)- $\alpha$-methylbenzylcarbamate] coated onto silica-gel. Reprinted with permission from [37]. Copyright (2003) American Chemical Society

$\mathrm{O}-\mathrm{H}$ group of ethyl lactate in turn serve as hydrogen acceptor and donor, respectively. The spectral shift of the $\mathrm{N}-\mathrm{H}$ bending mode of the chiral stationary phase (amide II) and the $\mathrm{C}=\mathrm{O}$ stretching mode of the lactate are larger for D- than for L-ethyl lactate. This shows that the $\mathrm{N}-\mathrm{H} \cdots \mathrm{O}=\mathrm{C}$ hydrogen bonding interaction is stronger in the case of Dethyl lactate. From relative band intensities for adsorbed D- and L-ethyl lactate a $\Delta \Delta \mathrm{G}$ of about $0.7 \mathrm{~kJ} / \mathrm{mol}$ can be estimated. Based on the above information a model for the relevant selector-selectand intermolecular interactions was proposed. More information on this system can be found in [37]. The technique presented above to probe chiral stationary phases can be applied to other types of chiral interfaces.

\section{Acknowledgements}

I am much indebted to former and present $\mathrm{PhD}$ students at ETH-Zürich for their enthusiasm and hard work. Particularly I thank Dr. Davide Ferri, Angelo Vargas, Ronny Wirz, Atsushi Urakawa, Norberto Bonalumi and Otmar Sonderegger. I furthermore thank my mentor Prof. Alfons Baiker, ETH-Zürich, for his generous support during my Habilitation. Prof. Urs Fringeli and Dr. Dieter Baurecht, University of Vienna, are thanked for many fruitful discussions on ATR spectroscopy and my new colleagues in Neuchâtel for helping me making the transition to Neuchâtel as smooth as possible. Support by the Swiss National Science Foundation through a SNF Förderungsprofessur is kindly acknowledged. The research described above was financially supported by the Swiss National Science Foundation and ETH-Zürich. Grants of computer time by ETH-Zürich and CSCS in Manno are acknowledged.

Received: July 14, 2003

[1] A. Baiker, Chimia 2001, 55, 796.

[2] B.M. Weckhuysen, J. Chem. Soc. Chem. Commun. 2002, 97.

[3] B.S. Clausen, H. Topsoe, R. Frahm, $A d v$. Catal. 1998, 42, 315.

[4] J.M. Thomas, Angew. Chem. Int. Ed. 1999 , $38,3589$.

[5] M. Hunger, J. Weitkamp, Angew. Chem. Int. Ed. 2001, 40, 2954.

[6] J. Ryczkowski, Catal. Today 2001, 68, 263.

[7] J.O.M. Bockris, S.U.M. Kahn, 'Surface electrochemistry: A molecular level approach', Plenum, New York 1993.

[8] F.M. Piras, A. Rossi, N.D. Spencer, Langmuir 2002, 18, 6606. 3, 2124. 123, 12074. $115,473$.

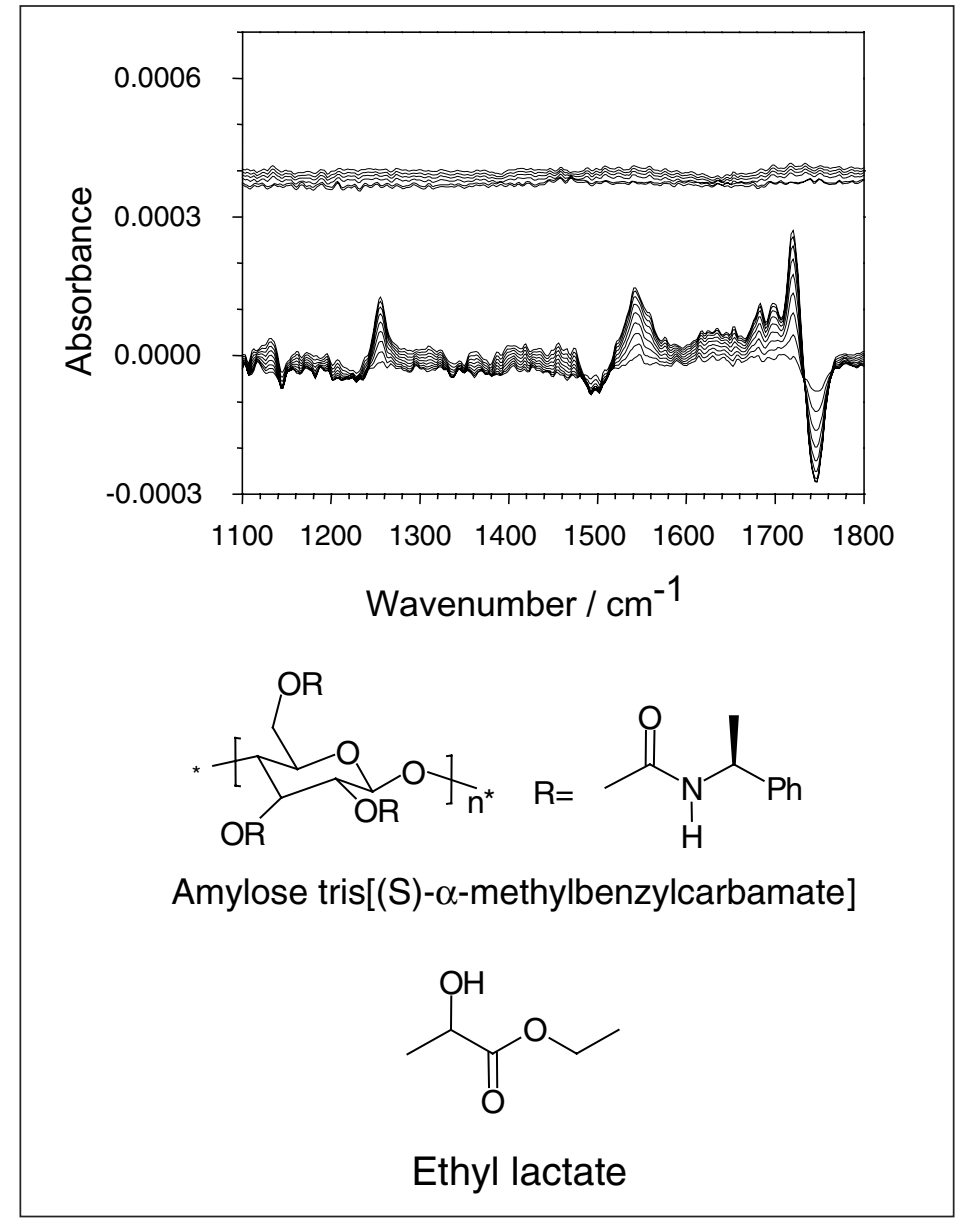

[9] C.M. Egglestone, W. Stumm, B. Sulzberger, M.D. Afonso, Geochim. et. Cosmochim. Acta 1998, 62, 585.

[10] U.P. Fringeli, D. Baurecht, H.H. Günthard, in 'Infrared and Raman Spectroscopy of Biological Materials', Eds. H.U. Gremlich, B. Yan, Dekker, New York / Basel 2000, p. 143.

[11] N.J. Harrick, 'Internal reflection spectroscopy', Interscience, New York 1967.

[12] T. Bürgi, A. Baiker, J. Phys. Chem. B 2002, 106, 10649.

[13] D. Baurecht, U. P. Fringeli, Rev. Sci. Instr. 2001, 72, 3782.

[14] T. Bürgi, Phys. Chem. Chem. Phys. 2001,

[15] D. Ferri, T. Bürgi, A. Baiker, J. Chem. Soc. Chem. Commun. 2001, 1172.

[16] D. Ferri, T. Bürgi, J. Am. Chem. Soc. 2001,

[17] D. Ferri, T. Bürgi, A. Baiker, J. Phys. Chem. B 2001, 105, 3187.

[18] D. Ferri, T. Bürgi, A. Baiker, Phys. Chem. Chem. Phys. 2002, 4, 2667.

[19] D. Ferri, T. Bürgi, A. Baiker, J. Catal. 2002, 210, 160.

[20] D. Ferri, T. Bürgi, A. Baiker, Helv. Chim. Acta 2002, 85, 3639

[21] C. Keresszegi, T. Bürgi, T. Mallat, A. Baiker, J. Catal. 2002, 211, 244.

[22] D. Ferri, S. Frauchiger, T. Bürgi, A. Baiker, J. Catal. 2003, 219, 425.

[23] A. Baiker, J. Mol. Catal. A: Chem. 1997,
[24] H.U. Blaser, H.P. Jalett, W. Lottenbach, M. Studer, J. Am. Chem. Soc. 2000, 122, 12675.

[25] O. Schwalm, B. Minder, J. Weber, A Baiker, Catal. Lett. 1994, 23, 271.

[26] K.E. Simons, P.A. Meheux, S.P. Griffiths, I.M. Sutherland, P. Johnston, P.B. Wells, A.F. Carley, M.K. Rajumon, M.W Roberts, A. Ibbotson, Recl. Trav. Chim. Pays-Bas 1994, 113, 465.

[27] A. Vargas, T. Bürgi, A. Baiker, J. Catal. 2001, 197, 378

[28] T. Bürgi, A. Baiker, J. Catal. 2000, 194, 445

[29] T. Bürgi, A. Vargas, A. Baiker, J. Chem. Soc. Perkin Trans. 2 2002, 1596.

[30] L.A. Nafie, T.A. Keiderling, P.J. Stephens, J. Am. Chem. Soc. 1976, 98, 2715. $48,357$.

[32] R.A. Sheldon, 'Chirotechnology', Marcel Dekker, New York 1993.

[33] N.M. Maier, S. Schefzick, G.M. Lombardo, M. Feliz, K. Rissanen, W. Lindner, K.B. Lipkowitz, J. Am. Chem. Soc. 2002, $124,8611$.

[34] B. Beden, C. Lamy, 'Spectroelectrochemistry', Plenum, New York 1989.

[35] Y.R. Shen, Annu. Rev. Phys. Chem. 1989 40, 327.

[36] W. Hug, Chimia 1994, 48, 386.

[37] R. Wirz, T. Bürgi, A. Baiker, Langmuir 2003, 19, 785 .
[31] L.A. Nafie, Ann. Rev. Phys. Chem. 1997, 